\title{
REGISTRO PALINOLÓGICO DE DEPÓSITOS SEDIMENTARES NEOPLEISTOCÊNICOS DO RIO MADEIRA, RONDÔNIA, AMAZÔNIA BRASILEIRA
}

\author{
Maria Ecilene Nunes da Silva MENESES \\ Etiene Fabbrin PIRES \\ Átila Augusto Stock DA ROSA \\ Luciano Artemio LEAL \\ Laís Aguiar da Silveira MENDES \\ Milton José de PAULA \\ Francisco Edinardo Ferreira de SOUZA \\ José Rafael Wanderley BENÍCIO \\ Leomir dos Santos CAMPOS
}

\begin{abstract}
RESUMO
O presente trabalho apresenta um estudo de identificação das assembléias palinológicas de uma camada de sedimentos numa área atualmente dominada por floresta, localizada na Bacia Hidrográfica do Alto Curso do Rio Madeira, na região de influência do Aproveitamento Hidrelétrico (AHE) Jirau, Estado de Rondônia, Amazônia Brasileira. Datações radiométricas pelo método do radiocarbono (AMS) indicam que o registro da camada compreende o intervalo entre 41.350 e 43.500 anos Antes do Presente (A.P.), correspondente ao Neopleistoceno. Os resultados mostram um conjunto de palinomorfos marcado por baixa diversidade polínica, essencialmente dominado por elementos de floresta tropical, e com aumento de tipos polínicos característicos de ambientes úmidos em direção ao topo da camada, como é o caso de Ludwigia, sugerindo provavelmente o desenvolvimento de um brejo. De forma geral, os dados mostram concordância com registros palinológicos de outras áreas estudadas na região da Floresta Amazônica, que indicam a estabilidade do ambiente florestal durante o mesmo período.
\end{abstract}

Palavras-chave: Palinologia, Neopleistoceno, vegetação, Estado de Rondônia, Amazônia Brasileira.

\section{ABSTRACT}

The present paper presents a study of identification of palynological assemblages from a layer of sediment taken from a modern forested area situated in the upper course of the Madeira River, within the region of influence of the AHE Jirau, Rondônia, Brazilian Amazon. Radiometric carbon dating using ${ }^{14} \mathrm{C}$ (AMS method) furnished ages between 41,350 and 43,500 years Before Present (B.P.), or Late Pleistocene, for the studied layer. The results reveal a set of palynomorphs marked by low diversity of pollen families with predominantly tropical forest types showing an upward increase in pollen grains typical of wet environments, probably suggesting the installation of a swamp. No other vegetational change was observed during the period recorded in this study, and in general the data are in agreement to palynological records obtained from other areas of the Amazon forest region that indicate a steady forested environment for the same period.

Keywords: Palynology, Late Pleistocene, vegetation, Rondônia state, Brazilian Amazon. 


\section{INTRODUÇÃO}

A palinologia constitui uma ferramenta amplamente utilizada para o reconhecimento dos tipos de vegetação, ambientes e climas que vigoraram no passado. A Amazônia corresponde a uma extensa região atualmente dominada por florestas, porém, rica em outras formações vegetais. Apesar dos múltiplos esforços, os estudos palinológicos nessa região ainda são escassos e pontuais para se compreender as mudanças ambientais e climáticas vigentes ao longo do Holoceno e, principalmente do Pleistoceno, cujos registros são ainda mais raros.

No âmbito dos trabalhos que contemplam o período do Pleistoceno destaca-se o estudo pioneiro de ABSY et al. (1991), que reconheceram períodos de retração da floresta e sua substituição por savana na Serra dos Carajás durante o Neopleistoceno, inferidos pelo aumento de ervas de Poaceae, Asteraceae, Borreria e Cuphea, típicas de ambientes savânicos. Segundo esses autores, tais períodos estiveram sob influência de um paleoclima mais seco que o atual.

Por outro lado, dados palinológicos obtidos por COLINVAUX et al. (1996) e BUSH et al. (2004) na região do Morro dos Seis Lagos (noroeste da Amazônia) mostraram a presença contínua da cobertura florestal durante o Neopleistoceno e Holoceno. Estes autores, entretanto, também registraram a presença de elementos adaptados a climas frios, como Ilex e Hedyosmum, o que evidencia a dominância de um clima mais frio que o atual durante o Neopleistoceno. Tais resultados levaram os referidos autores a argumentarem contra a teoria dos refúgios florestais proposta por HAFFER (1969), segundo a qual a floresta tropical amazônica teria sofrido fragmentação durante os períodos glaciais, sendo reduzida a ilhas ou refúgios de florestas úmidas em meio ao domínio de savanas herbáceas e arbustivas, vegetação adaptada a condições climáticas mais secas. Diante desta controvérsia, há ainda outros estudos que sugerem que a região amazônica permaneceu florestada durante o último ciclo glacial, embora tenha havido expansão de savanas nas regiões periféricas (MAYLE et al. 2009). Dessa forma, ainda não há um consenso geral entre a comunidade de pesquisadores acerca de como eram realmente as paisagens amazônicas durante o último ciclo glacial. No entanto, muitos acreditam que diversas regiões apresentaram diferentes condições climáticas que afetaram de modo diferenciado os ecossistemas amazônicos (COLINVAUX et al. 2000, BEHLING \& HOOGHIEMSTRA 2001, BUSH et al. 2004, BURBRIDGE et al. 2004).
É neste contexto que se insere o presente trabalho, cujo objetivo é contribuir para a identificação de paleoambientes na região amazônica e reconstituição das mudanças ambientais e climáticas ocorridas ao longo do Quaternário tardio, tendo como base a identificação das assembléias de grãos de pólen e esporos de suas paleofloras. O presente estudo foi realizado no canteiro de obras do Aproveitamento Hidrelétrico (AHE) Jirau, no Estado de Rondônia. Estudos dessa natureza haviam sido realizados somente ao longo de um trecho de $250 \mathrm{~km}$ entre a cidade de Porto Velho e a região de Humaitá (sul do Amazonas), numa área de ecótono savanafloresta, e se restringiam ao Holoceno (PESSENDA et al. 1998). A partir do trabalho conjunto de um grupo de pesquisadores do Programa de Investigação, Salvamento e Monitoramento Paleontológico do AHE Jirau, foi possível coletar amostras de sedimentos em um perfil exposto pelas escavações.

\section{MATERIAIS E MÉTODOS}

O canteiro de obras do AHE Jirau está localizado nas imediações da Ilha do Padre, alto curso do Rio Madeira, a cerca de $120 \mathrm{~km}$ a sudoeste da cidade de Porto Velho, Rondônia (Figura 1). O clima deste estado é do tipo equatorial (quente e úmido), com médias anuais de temperatura superiores a $26{ }^{\circ} \mathrm{C}$, e índice de precipitações elevado (entre 2.000 e $3.000 \mathrm{~mm}$ anuais), sobretudo entre os meses de dezembro a maio. A vegetação atual é caracterizada pela floresta tropical úmida, com manchas de cerrado. Especificamente, na região de abrangência do AHE Jirau, a vegetação é típica da floresta amazônica.

As amostras utilizadas para este estudo foram coletadas em um perfil exposto pelas escavações, no ponto denominado Estaca 93 (Figura 1), local de encontro do eixo da barragem com a casa de força, na margem direita do Rio Madeira, durante o mês de julho de 2011.

Um total de 10 amostras foram coletadas em intervalos fixos de $10 \mathrm{~cm}$ ao longo de uma camada de argila orgânica de $95 \mathrm{~cm}$ para datação e análises palinológicas. Para as datações foram retiradas duas amostras, respectivamente, da base e do topo da camada. As datações por radiocarbono foram realizadas com a técnica de espectroscopia de aceleração de massa (AMS) no laboratório da Beta Analytic, Flórida, EUA. As idades $\mathrm{C}^{14}$ obtidas foram calibradas em anos antes do presente (A.P.) a partir do software CALIB 6.0 (STUIVER \& REIMER 1993), utilizando-se a curva de calibração para o Hemisfério Sul. 

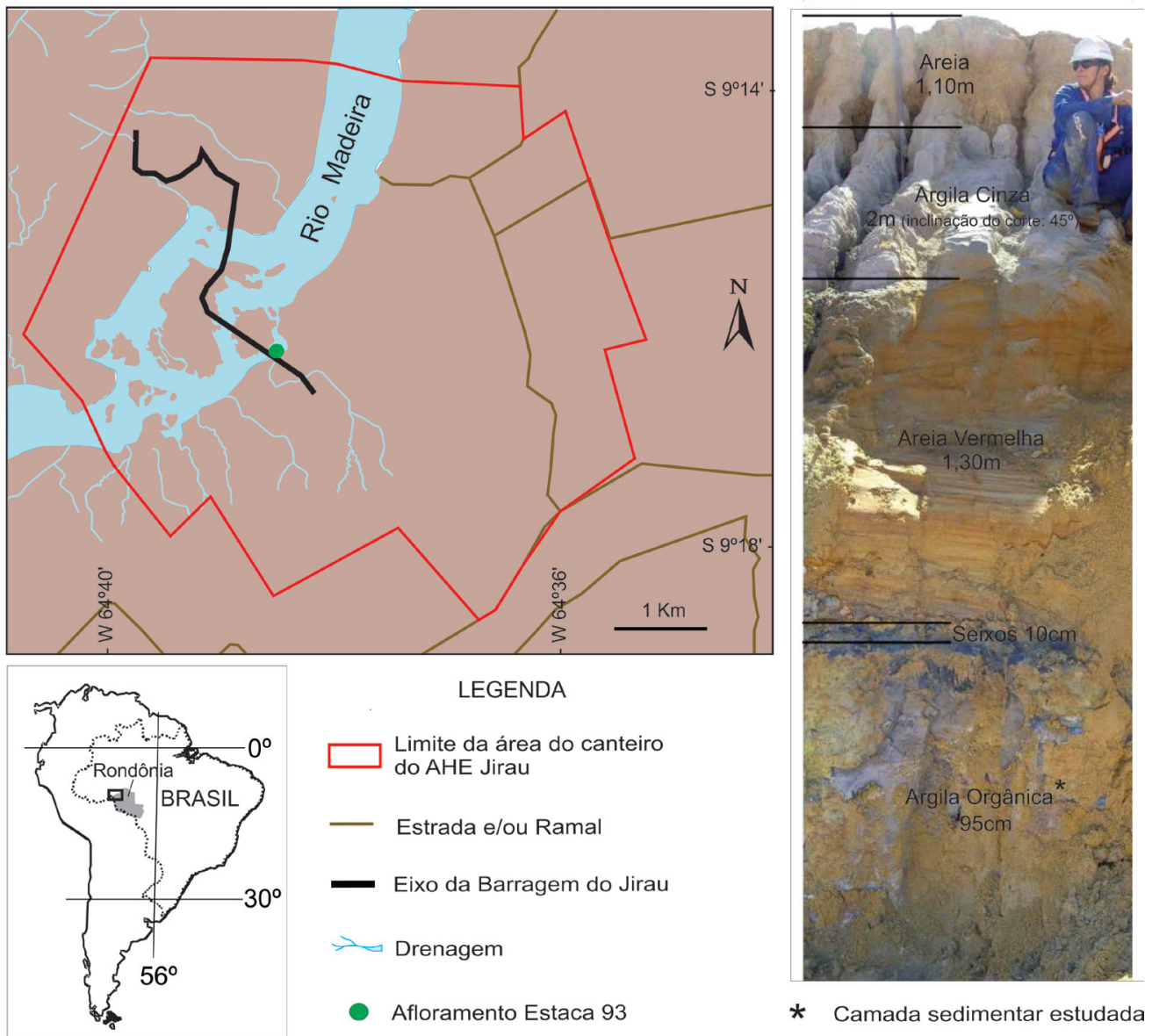

FIGURA 1 - Localização geográfica do canteiro de obras do AHE Jirau e do afloramento Estaca 93. A camada de argila orgânica amostrada para estudo palinológico é indicada pelo asterisco na foto do afloramento.

As análises palinológicas consistiram na identificação e quantificação dos grãos de pólen e esporos contidos nas amostras e foram realizadas nos Laboratórios de Paleobiologia, Genética e Bioquímica da Universidade Federal do Tocantins - UFT, Campus de Porto Nacional.

Para estas análises foram utilizadas pastilhas de sedimentos com cerca de $2 \mathrm{~cm}^{3}$, subamostradas de cada amostra. Estas foram submetidas ao tratamento químico padrão com a finalidade de obter como elemento residual somente grãos de pólen e esporos, conforme método adotado por FAEGRI \& IVERSEN (1989). Um tablete de Lycopodium clavatum foi adicionado a cada amostra a fim de se calcular a concentração de grãos de pólen e de esporos nos sedimentos (grãos $/ \mathrm{cm}^{3}$ ). O tratamento químico envolveu ataque com ácido clorídrico (HCL) para eliminação de possíveis carbonatos $\left(\mathrm{CO}_{3}\right)$ presentes no sedimento, ácido fluorídrico (HF) para eliminação dos silicatos e, finalmente, tratamento com acetólise (ERDTMAN 1952), para a remoção da celulose e polissacarídeos das amostras por oxidação. Uma vez que o material processado já se encontrava com tonalidades claras, não houve necessidade de submetê-lo ao tratamento com hidróxido de potássio $(\mathrm{KOH})$, normalmente usado para auxiliar na eliminação de matéria orgânica. A partir do material residual foram preparadas lâminas semi-permanentes utilizando-se gelatina glicerinada. A identificação taxonômica dos palinomorfos foi realizada com auxílio de bibliografia especializada, mais especificamente, ABSY (1979), ROUBIK \& MORENO (1991) e COLINVAUX et al. (1999).

Para calcular e plotar os diagramas polínicos foi utilizado o softwares TILIA e TILIAGRAPH (GRIMM 1987), programa de uso licenciado. Neste programa foi realizada uma análise de cluster (programa CONISS). 


\section{RESULTADOS}

A camada analisada é constituída da base até o topo por sedimento argiloso de coloração preta, compactado, com $95 \mathrm{~cm}$ de espessura, e é recober- ta por um pacote de sedimentos areno-sílticos pedogenizados de coloração amarela (Figura 1). As idades estão compreendidas entre o intervalo de 41.350 a 43.500 anos calibrados A.P. (Neopleistoceno), conforme tabela 1 .

TABELA 1 - Idades convencionais e calibradas para a base e o topo da camada de argila estudada

\begin{tabular}{cccccc}
\hline $\begin{array}{c}\text { Código do } \\
\text { laboratório }\end{array}$ & Amostras & $\begin{array}{c}\text { Profundidade } \\
(\mathrm{cm})\end{array}$ & $\begin{array}{c}\text { Idade C } \text { Convencional }^{4} \\
\text { Conve }\end{array}$ & $\begin{array}{c}\text { Idade Calibrada } \\
\text { anos AP (Sigma 2) }\end{array}$ & Erro \\
\hline Beta - 312037 & Estaca 93 base & $90-95$ & 43.500 & 43.500 & $+/-290$ \\
Beta - 312038 & Estaca 93 topo & $0-05$ & 36.200 & 41.350 & $+/-290$ \\
\hline
\end{tabular}

Foram identificados 62 tipos polínicos pertencentes a 30 famílias nas 10 amostras analisadas. Cerca de 7 tipos não foram identificados. Os tipos polínicos e esporos mais abundantes são ilustrados na figura 2. A figura 3 apresenta o diagrama palinológico de porcentagens dos principais tipos e famílias ao longo do perfil. O registro dos grupos ecológicos, a soma polínica, bem como os valores de concentração (grãos/ $\mathrm{cm}^{3}$ ) e de influxo (grãos/ $\mathrm{cm} / \mathrm{ano}$ ) são apresentados na figura 4.

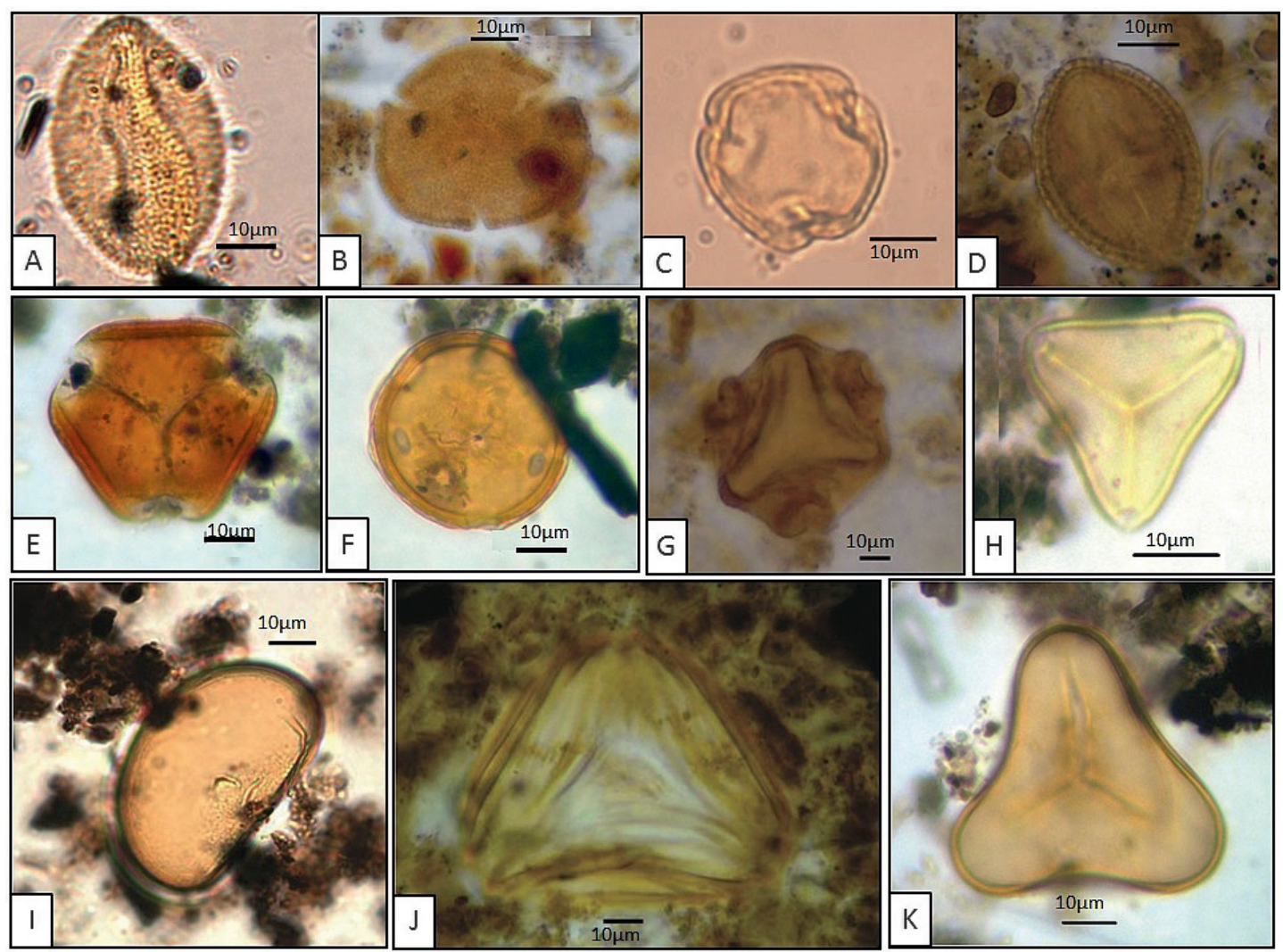

FIGURA 2 - Fotomicrografias dos principais tipos polínicos e esporos identificados nas amostras. A Bignoniaceae, B - Bombacaceae, C - Euphorbiaceae (Alchornea), D - Euphorbiaceae, E - Fabaceae, F - Malpighiaceae, G - Onagraceae (Ludwigia), H - Sapindaceae, I - Monolete psilado, J - Parkeriaceae (Ceratopteris), K - Trilete psilado. 


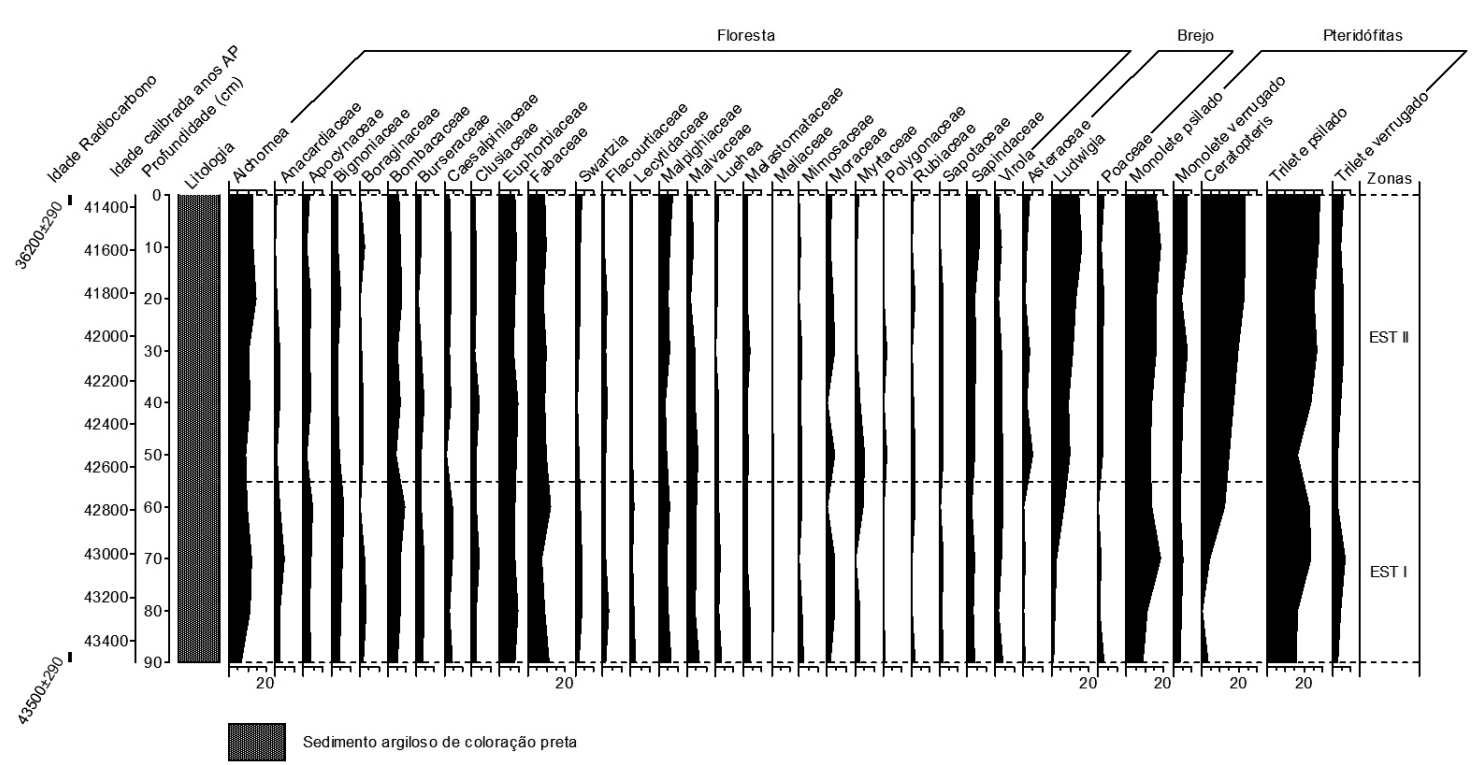

FIGURA 3 - Diagrama palinológico de porcentagem, mostrando os principais tipos e famílias, agrupados em floresta, brejos e pteridófitas.

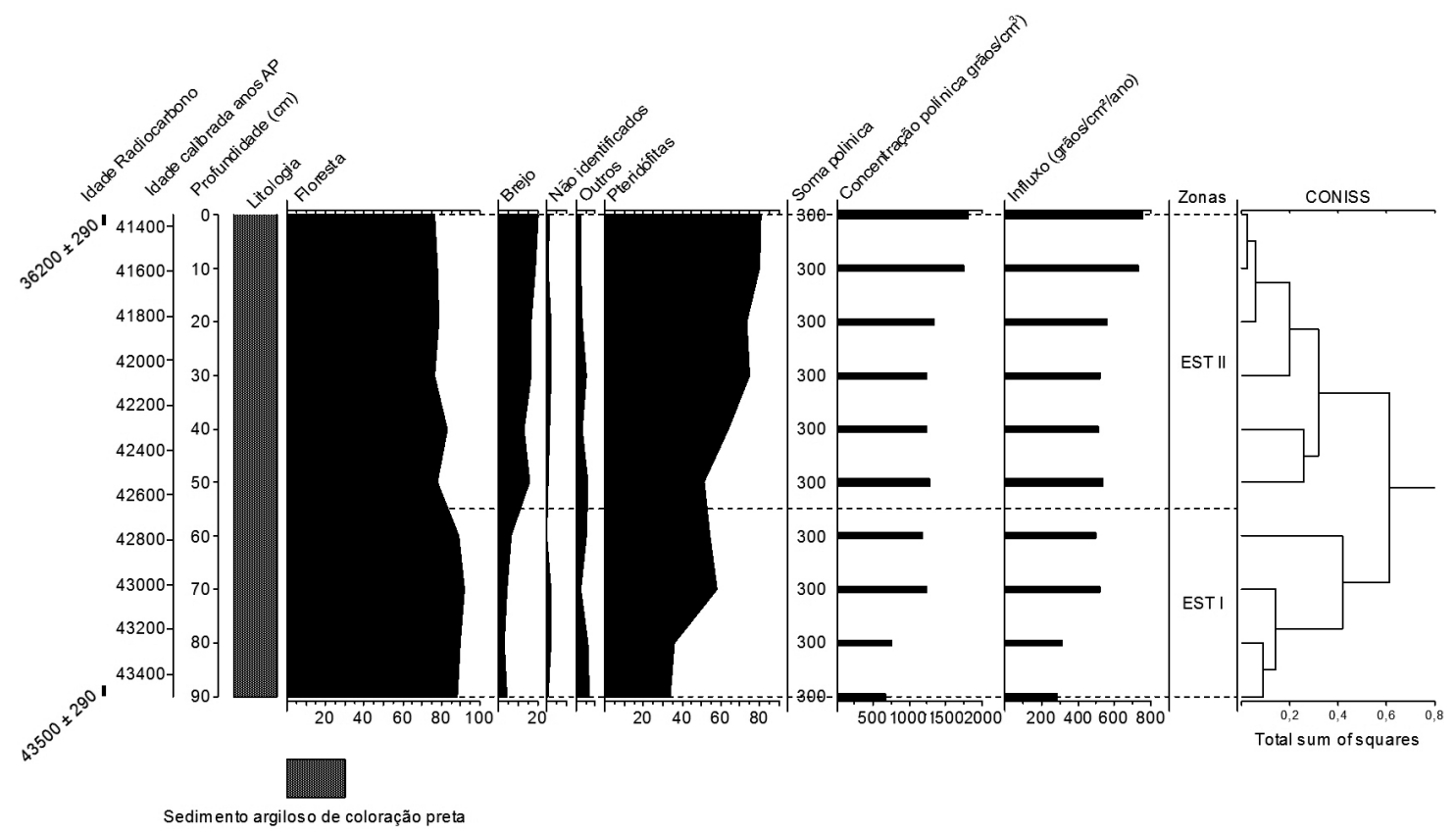

FIGURA 4 - Diagrama palinológico mostrando as somas dos grupos ecológicos, idades radiocarbono e calibradas, litologia, valores de concentração e influxo polínicos e zonas palinológicas.

A análise de cluster baseada na ocorrência dos palinomorfos de ambiente terrestre permitiu reconhecer duas zonas palinológicas: EST-I e EST-II.

A Zona EST-I (90-60 cm; 43.500-42.800 cal. anos A.P.) é marcada pela alta representação de elementos de floresta (88-92\%), com destaque para Alchornea (6-12\%), Fabaceae (6-12\%), Euphorbiaceae (7-9\%), Bombacaceae (5-9\%), Malpighiaceae (4-6\%), Malvaceae (4-6\%) e Bignoniaceae (3-6\%). Demais famílias representativas de floresta ocorrem em valores mais 
baixos $(<4 \%$ cada). O grupo ecológico de brejo apresenta baixos valores (2-6\%), tendo como elemento principal o gênero Ludwigia, que varia de 1 a $6 \%$, aumentando em direção à parte superior desta zona. Os valores de esporos de pteridófitas variam de 33 a $58 \%$, sendo os triletes psilados (15-24\%) os mais abundantes, seguidos pelos monoletes psilados (8-18\%) e Ceratopteris da família Parkeriaceae (1-12\%). Os valores de concentração polínica são estáveis nesta zona $\left(6,760-12,420\right.$ grãos $\left./ \mathrm{cm}^{3}\right)$, assim como os valores de influxo (282-519 grãos/ $\mathrm{cm}^{2} / \mathrm{ano}$ ).

A Zona EST-II $(50-0 \mathrm{~cm}$; 42.600-41.350 cal. anos A.P.), semelhantemente à zona anterior, é caracterizada pela abundância relativa de grãos de pólen de floresta (77-83\%), devido à contribuição dos mesmos táxons, a saber, Alchornea (9-14\%), Fabaceae (8-10\%), Euphorbiaceae (7-10\%), Bombacaceae (4-7\%), Malpighiaceae (3-7\%), Sapindaceae (3-6\%), Bignoniaceae (3-5\%) e Malvaceae (2-5\%). Entretanto, os valores percentuais de pólen de floresta apresentam-se ligeiramente decrescentes em comparação à zona EST-I, devido ao aumento relativo verificado para o grupo de brejos (13 a 20\%), em especial dos grãos de pólen de Ludwigia (9-16\%). Poaceae e Asteraceae, que foram incluídas neste grupo, ocorrem em porcentagens mais baixas $(<5 \%)$. O grupo de pteridófitas apresenta valores entre 51 e $80 \%$, sendo, portanto, maiores que na zona anterior, aumentando progressivamente em direção ao topo da camada, especialmente Ceratopteris (14-23\%), seguidos de triletes psilados (16-28\%) e monoletes psilados (12-18\%).

A concentração polínica é maior nesta zona $\left(12,300-18,000\right.$ grãos $\left./ \mathrm{cm}^{3}\right)$, assim como os valores de influxo (534-753 grãos/ $\mathrm{cm}^{2} / \mathrm{ano}$ ).

\section{DISCUSSÃO E CONCLUSÕES}

A dominância de grãos de pólen das famílias polínicas Alchornea, Fabaceae, Euphorbiaceae, Bombacaceae, Malpighiaceae, Bignoniaceae, Malvaceae e Sapindaceae indica um ambiente local florestado, uma vez que a maioria destes tipos polínicos são zoófilos, ou seja, não percorrem grandes distâncias como os grãos polinizados pelo vento, representando assim a vegetação local e circundante. A presença de Alchornea, que é um gênero pioneiro, pode ainda sugerir tratar-se de um ambiente perturbado naturalmente sob regeneração.

Este ambiente, provavelmente um lago de planície aluvionar circundado por floresta, foi se tornando cada vez mais raso devido ao processo natural de colmatação, conforme sugerido pelo au- mento expressivo de pteridófitas, que podem ter colonizado o local em função da diminuição da lâmina d'água. Além disso, o aumento de grãos de pólen de Ludwigia da família Onagraceae em direção ao topo da camada indica possível desenvolvimento local de um brejo ou outro ambiente de águas rasas, uma vez que este gênero, seja na forma de arbusto ou macrófita aquática, adapta-se bem a estes tipos de habitat. O aumento de Ceratopteris (Parkeriaceae) em direção ao topo da camada reforça essa interpretação, por se tratar de uma pteridófita típica de ambientes brejosos tropicais.

Ao contrário de estudos realizados em outros locais da região amazônica sobre o Neopleistoceno, os dados obtidos neste trabalho não confirmaram a presença de tipos polínicos adaptados a climas mais frios (ex. Hedyosmum, Ilex, etc), tais como os encontrados nos sedimentos da Lagoa da Pata, extremo noroeste da Amazônia (BUSH et al. 2004). Também não foram identificados grãos de pólen exóticos como Alnus, proveniente dos Andes, que poderia alcançar a área de estudo através do transporte fluvial. De forma geral, pode-se inferir que o registro polínico desta área no período em questão representa a vegetação local. Além disso, está em conformidade com a vegetação moderna da região, atualmente encontrada na flora ciliar do rio Madeira. Entretanto, é conveniente ressaltar que os dados aqui apresentados são lateral e temporalmente descontínuos. Somente com a aquisição de mais informações, provenientes de outros locais, será possível a reconstituição das mudanças ambientais e climáticas na bacia do Rio Madeira, de forma a contribuir para o conhecimento da dinâmica das paisagens amazônicas e da América do Sul.

\section{AGRADECIMENTOS}

Os autores agradecem à Energia Sustentável do Brasil S.A., pelo apoio dado para a apresentação de trabalhos e liberação dos dados para publicação, aos editores e aos revisores anônimos, pelas correções e melhorias sugeridas no manuscrito original.

\section{REFERÊNCIAS BIBLIOGRÁFICAS}

ABSY, M.L. 1979. Palynological study of Holocene sediments in the Amazon Basin. University of Amsterdam, Amsterdam, Thesis, 102 p.

ABSY, M.L.; CLEEF A.L.M.; FOURNIER, M.; MARTIN, L.; SERVANT, M.; SIFEDDINE, A.; DA SILVA, M.F.; SOUBIËS, F.; SUGUIO, K.; TURCQ, B.; VAN DER HAMMEN, T. 1991. Mise en évidence de quatre phase d'ouverture de la forêt dense dans le sudest 
del'Amazonie au cours des 60000 dernières années. Premiére comparaison avec d sautres régions tropicales. C.R. Académie Science Paris, 312: 673-678.

BEHLING, H.; HOOGHIEMSTRA, H. 2001. Neotropical savanna environments in space and time: Late Quaternary interhemispheric comparisons. In: V. Markgraf (ed.) Interhemispheric Climate Linkages, Academic Press, p. 307-323.

BURBRIDGE, R.E.; MAYLE, F.E.; KILLEEN, T.J. 2004. Fifty-thousand-year vegetation and climate history of Noel Kempff Mercado National Park, Bolivian Amazon. Quaternary Research, 61: 215-230

BUSH, M.B.; DE OLIVEIRA, P.E.; COLINVAUX, P.A.; MILLER, M.C.; MORENO, J.E. 2004. Amazonian paleoecological histories: one hill, three watersheds. Palaeogeography, Palaeoclimatology, Palaeoecology, 214: 359-393.

COLINVAUX, P.A.; DE OLIVEIRA, P.E.; MORENO, J.E.; MILLER, M.C.; BUSH, M.B. 1996. A long pollen record from lowland Amazonia: forest and cooling in glacial times. Science, 247: 85-88.

COLINVAUX, P.A.; DE OLIVEIRA, P.E.; PATINO, J.E.M. 1999. Amazon Pollen Manual and Atlas. Harwood Academic Publishers, Singapore, $330 \mathrm{p}$.

COLINVAUX, P.A.; DE OLIVEIRA, P.E.; BUSH, Y.M.B. 2000. Amazonian and neotropical plant communities on glacial time-scales: the failure of the aridity and refuge hypotheses. Quaternary Science Reviews 19: 141-169.
ERDTMAN, G. 1952. Pollen morphology and plant taxonomy: Angiosperms. Almqvist \& Wiksell, Stockholm, 539 p.

FAEGRI K.; IVERSEN, J. 1989. Textbook of Pollen Analysis. John Wiley \& Sons, New York, $328 \mathrm{p}$.

GRIMM, E.C. 1987. CONISS: A Fortran 77 program for stratigraphically constrained cluster analysis by the method of the incremental sum of squares. Computer and Geosciences, 13: 13-35.

HAFFER, J. 1969. Speciation in Amazonian forest birds. Science, 165: 131-37.

MAYLE, F.E.; BURN, M.J.; POWER, M.; URREGO, D.H. 2009. Vegetation and fire at the Last Glacial Maximum in tropical South America. In: F. Vimeux, F. Sylvestre \& M. Khodri (eds.) Past Climate Variability in South America and Surrounding Regions From the Last Glacial Maximum to the Holocene. Springer Science Business Media B. V, p. 89-112.

PESSENDA, L.C.R.; GOMES, B.M.; ARAVENA, R.; RIBEIRO, A.S.; BOULET, R.; GOUVEIA, S.E.M. 1998. The carbon isotope record in soils along a forest-cerrado ecosystem transect: implication for vegetation changes in Rondônia State, southwestern Brazilian Amazon region. The Holocene, 8: 631-635.

ROUBIK, D.W.; MORENO, J.E. 1991. Pollen and spores of Barro Colorado Island. Missouri Botanical Garden, 36, 270 p.

STUIVER, M.; REIMER, P.J. 1993. Extended 14C database and revised CALIB radiocarbon calibration program. Radiocarbon, 35: 215-230.

\section{Endereço dos autores:}

Maria Ecilene Nunes da Silva Meneses, Laís Aguiar da S. Mendes, Milton José de Paula, Francisco Edinardo Ferreira de Souza e José Rafael Wanderley Benício - Universidade Federal do Tocantins, Rua 15, Quadra 12, s/n, Setor Jardim dos Ipês, CEP 77500-000, Porto Nacional, TO.E-mails: mariaecilene@ yahoo.com.br, laisasmendes@gmail.com, miltonuft@yahoo.com.br, edinardo.souza@yahoo.com.br, rafaeljwbenicio@yahoo.com.br

Etiene Fabbrin Pires - Universidade Federal do Tocantins, Rua Teotônio Segurado, $\mathrm{n}^{\circ}$ 150, Setor Jardim Brasília, CEP 77500-000, Porto Nacional, TO. E-mail: etienefabbrin@uft.edu.br 
Átila Augusto Stock Da Rosa - Universidade Federal de Santa Maria, Avenida Roraima, 1000, Camobi, CEP 97105-900, Santa Maria, RS.E-mail: atila@smail.ufsm.br

Luciano Artêmio Leal e Leomir dos Santos Campos - Universidade Estadual do Sudoeste da Bahia, Avenida José Moreira Sobrinho, S/N, Jequiezinho, CEP 45.206-190, Jequié, BA. E-mails: luciano.artemio@, gmail.com, leomirsxc@yahoo.com.br

Artigo submetido em 10 de janeiro de 2013, aceito em 2 de outubro de 2013. 